\title{
Simple ultrasound evaluation of fetal adrenal glands as a marker of imminent preterm birth: a preliminary report
}

\author{
Gabor M, Kunochova I, Krizko M Jr, Alfoldi M, Feriancova M, Ferianec V \\ 2nd Department of Gynecology and Obstetrics, Faculty of Medicine, Comenius University, Bratislava, Slovakia. \\ ferianec@gmail.com
}

\begin{abstract}
OBJECTIVE: We present a simple 2D big ultrasound measurement of the fetal adrenal glands and their biometrical growth analysis highlighted as a potential preterm birth marker.

METHODS: This was a prospective observational case-control study. Sixty-four patients were included in the study (32 with the diagnosis of imminent preterm birth before 37 and 32 controls) from January 2018 to May 2020. Anteroposterior dimensions and circumferences of the whole adrenal glands and their central zones were measured by simple B-mode ultrasound imaging. For the statistical analysis, StatsDirect 3.0 and ROC curves were used. As a studied descriptor, routine standard ultrasound cervical measurements were added (cervical length, funneling, sludge, cervical dilatation, and cervical glandular area).

RESULTS: In biometrics of gestational age-related changes, a significant analysis of the overall growth of the adrenal gland was observed (circumference $p<0.001$, anteroposterior diameter $p=0.02$ ). The growth of the central zone was observed independently of gestational age. The growth of the central zone of the fetal adrenal glands revealed significant changes between the group of patients who delivered prematurely and control groups $(p<0.01)$. The ideal cut-off value of the proportion of enlargement of the fetal zone as a predictor of preterm delivery before week 37 was $45.1 \%$, with the sensitivity of $87.5 \%$ and specificity of $85.4 \%$.

CONCLUSIONS: A simple 2D B-mode measurement of the fetal adrenal glands' central zone growth can be applied as an additional marker in the prediction of true preterm delivery. The natural biometrical overall growth of the adrenal glands seems to be dependent on gestational age, whereas that of the central adrenal gland zone seems to be independent on gestational age (Tab. 3, Fig. 5, Ref. 26). Text in PDF www.elis.sk KEY WORDS: preterm birth, prediction, ultrasound predictors, fetal adrenal glands.
\end{abstract}

\section{Introduction}

Preterm birth is a persistent perinatological problem despite the effort of its exact prediction. Modern perinatal medicine uses a spectrum of preterm birth predictive markers with defined specificity and sensitivity, but its exact clinical interpretation is still difficult (1).

The incidence of preterm births and vague clinical reliability of prediction define the need for new, additional markers to improve total sensitivity and specificity of the preterm delivery prediction (1).

Clinical symptoms and signs of preterm birth are applicable and have low sensitivity and specificity. Cervical sonography is widely used with good positive predictive values; biochemical markers like fetal fibronectin are applied with good negative predictive values. Recent studies also describe new morphological ultrasound markers of preterm birth, such as amniotic fluid sludge, cervical glandular area, and fetal adrenal glands measurement (2).

2nd Department of Gynecology and Obstetrics, Faculty of Medicine, Comenius University, Bratislava, Slovakia

Address for correspondence: V. Ferianec, Assoc Prof, MD, PhD, 2nd Department of Gynecology and Obstetrics, Faculty of Medicine, Comenius University, Ruzinovska 6, SK-826 06 Bratislava, Slovakia.

Phone: +421.905385670
In contrast to the published studies which use the 3D ultrasound evaluation of the fetal adrenal glands to study the correlation with preterm birth, this study evaluates simple 2D ultrasound measurement of the anteroposterior diameter and circumferences of fetal adrenal glands including their central zone $(3,4)$. The results describe the diagnostic principles and usability of this marker in order to encourage the other scientific teams to study this simple additional marker of preterm birth.

\section{Materials and methods}

The study was designed as a prospective, non-interventional, observational case-control study and was conducted during the period from January 2018 to May 2020 at University Hospital Bratislava. The patients were recruited after an interview with an understanding of the study principles, and all subjects gave their informed consent for inclusion before participating in the study. The study was conducted in accordance with the Declaration of Helsinki, and the protocol was approved by the Ethics Committee of the University Hospital Bratislava.

The primary selection included hospitalized patients in the maternal-fetal center with the diagnosis of imminent preterm birth - partus praematurus imminens (PPI). Strict criteria were applied to this primary group of various patients to achieve purity of the 
Tab. 1. Inclusion and exclusion criteria of the diagnosis of PPI.

\begin{tabular}{l} 
Inclusion criteria \\
Progression of the cervical score by digital palpation \\
Minimally 4 contractions of the uterus on a 20 -minute tocogram, also \\
subjectively described by the patient \\
Primigravidity \\
Gestational age $23+6$ to $37+6$ \\
The cervical length by transvaginal ultrasonography under $25 \mathrm{~mm}$ \\
(including) \\
Exclusion criteria \\
Previous preterm labor \\
Patients after cervical and uterine surgery \\
Congenital anomalies of the uterus \\
Uterine fibroids \\
Pathologies of the insertion or function of the placenta \\
Pathological amniotic fluid index \\
Congenital anomalies of the fetus and verified aneuploidy \\
Intrauterine growth retardation \\
Exogenous insult of trauma during gravidity \\
Premature rupture of membranes \\
Status after intrauterine invasive procedures \\
Cerclage \\
Retroplacental hematoma \\
Vaginal bleeding with medical history of imminent miscarriage \\
Preeclampsia, eclampsia, HELLP syndrome \\
Pathologies during pregnancy-hypertension, diabetes, hematologic diseases, \\
pregnancy after in vitro fertilization \\
Incomplete ultrasound diagnosis (i.e., inappropriate acoustic conditions) \\
\hline
\end{tabular}

\section{Tab. 2. Evaluated ultrasonographic parameters.}

Length of the cervix (mm)

Dilatation of the internal cervical os

Qualitative presence of the cervical glandular area

An echogenic dilatation of the cervical canal

Presence of the amniotic fluid sludge

studied group. The inclusion and exclusion criteria are in accord with the International Preterm Labor Council (IPLC). Their list is to be found in Table 1 .

All the recruited patients underwent standard diagnostic and therapeutic procedures, and additional measurements are as follows. Ultrasound was performed on a Zonare machine using standard $7 \mathrm{MHz}$ transvaginal and $4 \mathrm{MHz}$ convex transabdominal probe. The patients were diagnosed by the same sonographer. Ultrasonographic parameters were evaluated by using the transvaginal midsagittal scan listed in Table 2.

The fetal adrenal glands were depicted zoomed, and calipers were used to measure them in the transverse scan in B-mode. The maximal anteroposterior adrenal gland (APAG) diameter $(\mathrm{mm})$ of one adrenal gland of the fetus (Fig. 1) and maximal AP diameter of its central echogenic zone called anteroposterior fetal zone (APFZ) was measured 3 times (Fig. 2). In the same plane, the circumference values of the mentioned structures were measured 3 times using the ellipse: circumference of the whole adrenal gland (CIRC-AG) and circumference of the fetal zone (CIRC-FZ). All types of measurements are shown schematically in Figure 3. The arithmetic average and the percentage of the fetal zone of the whole gland diameter was calculated.

The date, time of the delivery, gestational week, and interval from ultrasound measurement to delivery was retrospectively verified by means of patients' documentation.

The studied group of patients with the diagnosis of imminent preterm birth (PPI group) was divided into the following subgroups, namely PP subgroup (partus praematurus) and PPI-C subgroup (first control group). The PP subgroup consisted of patients who delivered prematurely (up to $37+6$ gestational week, inclusively), whereas those who delivered at term, i.e., after $38+0$ gestational week, inclusively, were separated into the PPI-C subgroup. A second independent control group $\mathrm{C}$ was added - these were cases with physiological gravidities who met the study criteria and in whom the exclusion of the imminent preterm birth and term delivery had been verified.

Statistics were processed with the program StatsDirect 3.0. The correlation between the groups was analyzed through the simple linear regression, and Pearson's coefficient was used. The statistical significance of the changes was analyzed by one-factor analysis of variance (ANOVA) and Tukey post-hoc test (TukeyKramer multiple comparisons) with $\mathrm{p}<0.05$ considered significant.

As first in the process, the spread plot and box \& whisker plot were created followed by an analysis of variance. The results of evaluated markers were analyzed, ROC (receiver operating characteristic) curves were used, and sensitivity and specificity were defined. ROC curve definition of the optimal cut-off point is presented in Figure 4. The optimum cut-off point for the fetal zone enlargement as a predictor of preterm birth, predictive value of the positive test, posttest likelihood of disease, predictive value of negative test, posttest likelihood of no disease, posttest disease likelihood despite negative test and sensitivity and specificity confidence intervals were defined.

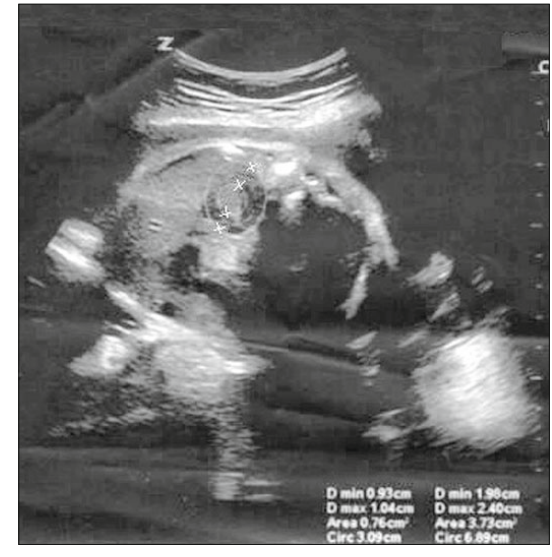

Fig. 1. Fetal adrenal gland in simple transverse plane - measured with calipers.

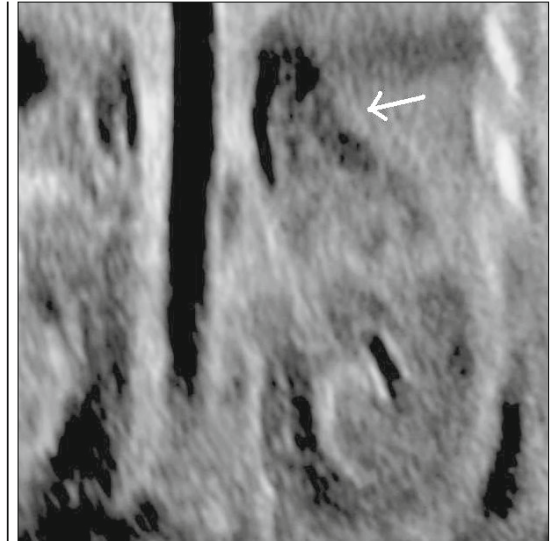

Fig. 2. Fetal adrenal gland ultrasound in frontal plane with a clearly defined central fetal zone (arrow). 


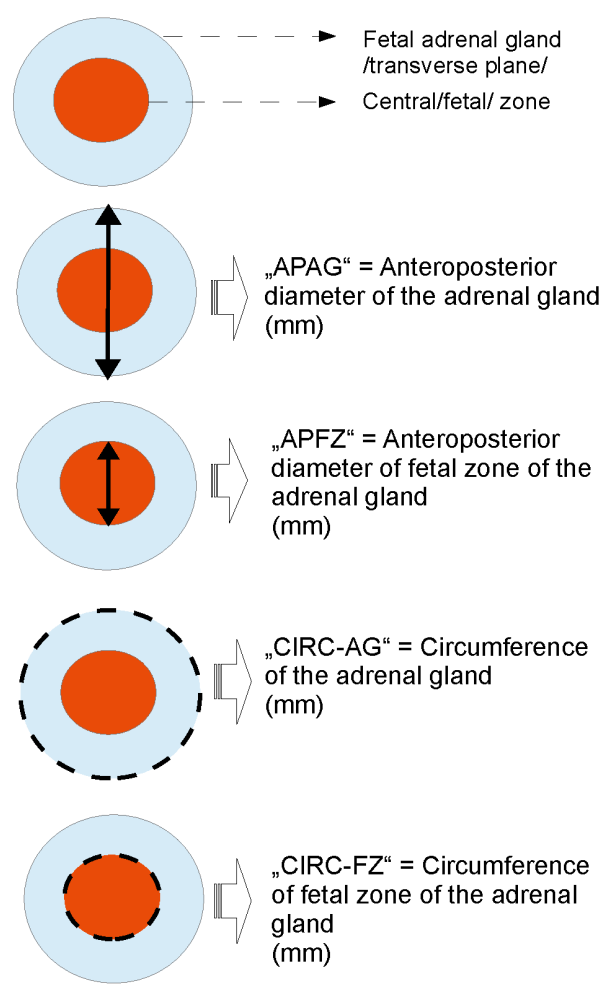

Fig. 3. Fetal adrenal gland ultrasound measurement.

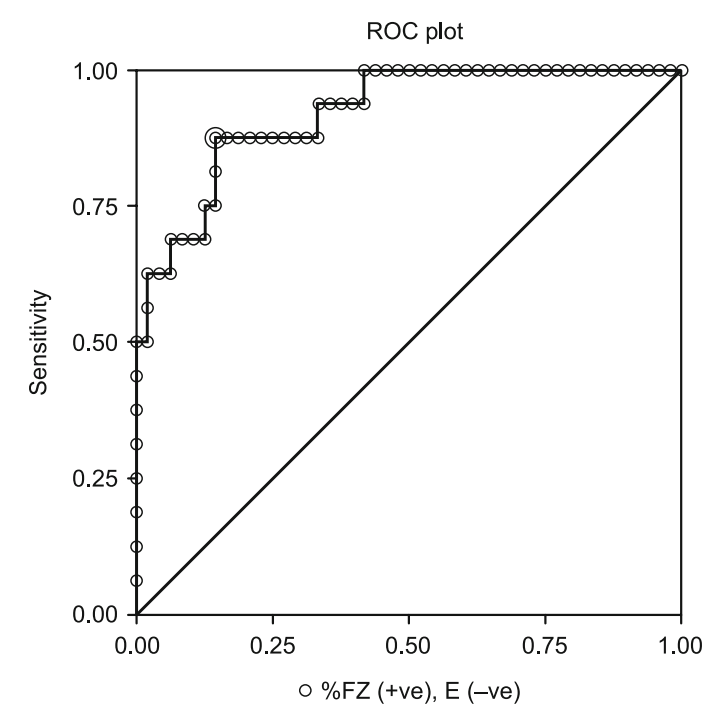

Data set: \% FZ (+ve), E (-ve) Area under ROC curve by extended trapezoidal rule $=0,920573$ Wilcoxon estimate of area under RoC curve $=0,920573$ Delong standard error $=0,036825: 95 \% \mathrm{Cl}=0,848397$ to 0,992748 Optimum cut-off point selected $=45,07$

Fig. 4. ROC curve definition of the optimal cut-off value for the percentage of central fetal zone enlargement as a preterm delivery predictor.

\section{Results}

Primary 214 patients were observed, of whom only 64 patients were measured in studied groups. According to strict study criteria, $148(81.3 \%)$ of patients could not be recruited. The main reasons for excluding the patients were as follows: false interpretation of the diagnosis of imminent preterm birth, absence of contractions on tocogram, history of cervical surgery (conization of the cervix), cervicometry over $25 \mathrm{~mm}$ and diseases of the mother.

Two patients of all those measured were excluded because of inappropriate acoustic conditions; these two patients were from the PPI group $\left(\mathrm{n}_{(\mathrm{PPI})}=34-2=32\right)$. After the final analysis of the PPI group, 18 patients became part of the group of patients with true preterm delivery $\left(\mathrm{n}_{(\mathrm{PP})}=18\right)$. The rest of the PPI group were separated into the first control group PPI-C $\left(\mathrm{n}_{(\mathrm{PPI}-\mathrm{C})}=14\right)$. The group $\mathrm{C}$ (second control group) consisted of cases with physiological graviditiy $(\mathrm{n}(\mathrm{C})=32)$. All this information is summarized in Table 3.

A proportion of $56.2 \%$ of patients from the whole PPI group delivered prematurely. As a description and introduction of the PPI group, results were obtained as follows: the average length of the cervix was $17.15 \mathrm{~mm}$, positivity of the funneling marker was in $46.9 \%$, cervical canal was dilated more than $5 \mathrm{~mm}$ in $34.3 \%$, and amniotic fluid sludge was positive in $21.9 \%$ of patients. The cervical glandular area (CGA) marker was positive in $21.0 \%$. The average estimated fetal weight (EFW) was $1,833 \mathrm{~g}$ with maximum of $2,850 \mathrm{~g}$ and minimum of $725 \mathrm{~g}$.

The measurements of fetal adrenal glands revealed average results as follows: APAG diameter of $19.6 \mathrm{~mm}$, the APFZ diameter of $9.1 \mathrm{~mm}$, CIRC-AG of $61.5 \mathrm{~mm}$, and CIRC-FZ of $30.3 \mathrm{~mm}$. We confirmed a significant correlation $(p=0.02)$ of the increase in APAG diameters and gestational age. The changes in APFZ were not significant $(p=0.06)$ in correlation with gestational age. The circumferences of the whole fetal adrenal gland correlated with the increase in gestational age more significantly than it was observed relative to its diameter $(p<0.001)$ In that sense, the circumference of the fetal zone did not correlate at all $(\mathrm{p}=0.32)$.

The most important part of the study was the description of the parameter of fetal zone growth of the adrenal gland in AP as a predictor of preterm birth. The percentage of the central fetal zone AP measurement revealed significant changes between the patients who delivered prematurely and control groups (subgroups PP, PPI$\mathrm{C}$, and $\mathrm{C})(\mathrm{p}<0.001)$ while the percentage varied unambiguously in the groups (Fig. 5). Those changes between control groups PPI$\mathrm{C}$ and $\mathrm{C}$ were not significant $(\mathrm{p}=0.227)$. The ideal cut-off value of the percentage (growth) of the AP diameter of the fetal zone as a marker of preterm delivery before week 37 was described using the ROC curves. At the percentage of $45.1 \%$, the sensitivity, specificity, positive predictive value, negative predictive value and likelihood of disease testing negative were $87.5 \%, 85.4 \%, 66.7 \%$, $95.3 \%$, and $4.6 \%$, respectively while the confidence intervals of reliability (95\%) for sensitivity and specificity were 61.6-98.4\% and $72.2-93.9 \%$, respectively.

\section{Discussion}

In the previous studies, Romero postulates that the application of preterm birth markers should not represent the standard screening for the normal not-at-risk population but only for selected 
Tab. 3. Group of included patients.

\begin{tabular}{|c|c|c|}
\hline Group & Name of the group / description & Number of the patients \\
\hline Primary observed patients & N0 & $\mathrm{N} 0=214$ \\
\hline Excluded patients & $\mathrm{Nx}$ & $\mathrm{Nx}=148$ \\
\hline \multirow{5}{*}{ Included patients } & $\mathrm{N} /$ all included patients/ & $\mathrm{N}=66$ \\
\hline & $\begin{array}{l}\text { PPI = patients with the diagnosis of imminent preterm labor } \\
-\mathrm{PP}=\text { partus prematurus } \\
\text { true premature delivery }\end{array}$ & $\begin{array}{l}N(P P I)=34 \\
N(P P)=18\end{array}$ \\
\hline & $\begin{array}{l}\text { PPI-C = partus prematurus imminens } \\
\text { control, patients with final delivery at term }\end{array}$ & $\mathrm{N}(\mathrm{PPI}-\mathrm{C})=14$ \\
\hline & Unmeasurable patients /Xx/ & $\mathrm{N} / \mathrm{Xx} /=2 /$ patients from the PPI group/ \\
\hline & $\mathrm{C}$ / control group, physiological gravidities, delivery at term/ & $\mathrm{N}(\mathrm{C})=32$ \\
\hline
\end{tabular}

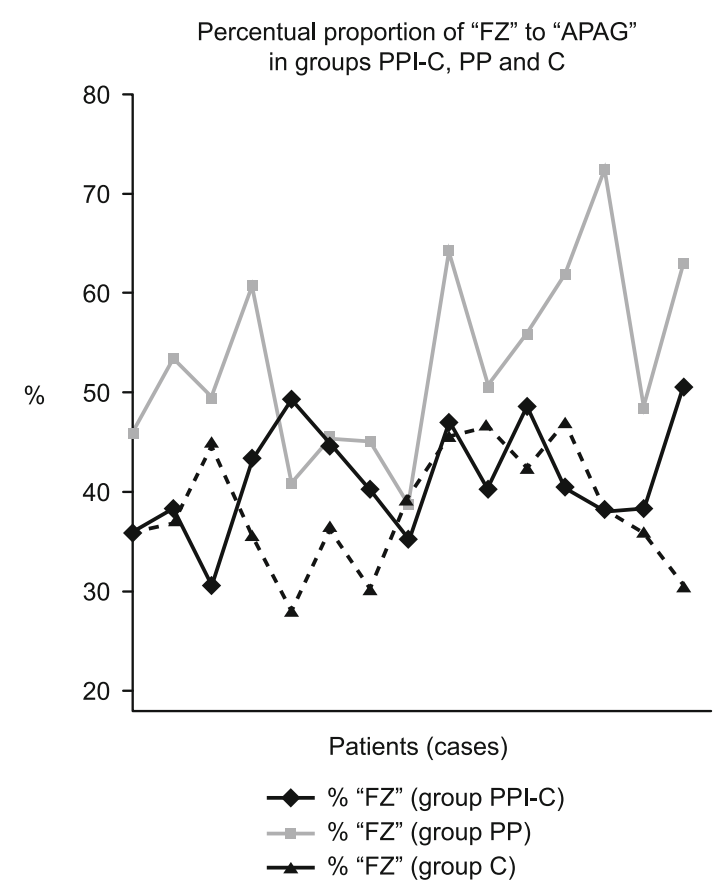

Fig. 5. Percent proportion of fetal zone (FZ) to anteroposterior adrenal gland (APAG) diameter in responsible groups PPI-C, PP and C.

high-risk groups (5). In our study, we applied a simple ultrasound marker of preterm birth to a purely selected group, which was at a high risk of preterm delivery.

The high percentage of patients excluded from the primarily selected patients (more than $80 \%$ ) definitely describes the clinical ambiguity of the diagnosis of imminent preterm birth and thus also the need for applying new markers for preterm delivery prediction by outpatient diagnosis. On the spectrum of exclusion criteria, it is possible to see the fact that the diagnosis of imminent preterm birth at the first gravidity entirely without any other maternal pathologies is relatively rare. This encourages Romero's parturition syndrome conception of preterm birth to be more like a single disease (2).

The average gestational week at the delivery in our studied PPI group was 30.7, which is in the range of high-risk prematurity. Using the mentioned strict criteria, we defined the high-risk group in which $56.2 \%$ of patients delivered prematurely.
Ultrasound cervicometry is a routine diagnostic method of imminent preterm birth. The average normal length of the cervix should be in the range of $32-46 \mathrm{~mm}$. Patients with cervical length of $25 \mathrm{~mm}$ should be strictly monitored as high-risk patients, while the length under $20 \mathrm{~mm}$ is definitely defined as cervical insufficiency. The risk for preterm birth when the cervical length is over $35 \mathrm{~mm}$ is low; at the length of $15 \mathrm{~mm}$, the risk rises exponentially, while the length of $5 \mathrm{~mm}$ represents a $44 \%$ probability of preterm birth. Another risk factor for preterm birth lies in the presence of funnel formation below the internal os and shortening of the residual cervical canal.

According to evidence-based medicine (EBM) studies, the incompetence of the cervix is associated with its length being under $20 \mathrm{~mm}$ or under $30 \mathrm{~mm}$ with the funneling more than $10 \mathrm{~mm}$ deep. By the depth of $25 \%$ of the funnel, the risk of prematurity is approximately $10 \%$, by that of $25-50 \%$, the risk is $30 \%$, and by $50 \%$ or more, the premature birth occurs in $75 \%$ of cases $(6-11)$.

In our studied PPI group, the average length of the cervix was $17.15 \mathrm{~mm}$, the positivity of the funneling marker was up to $46.9 \%$, the cervical canal was dilated up to $34.4 \%$.

Another ultrasound sign reflecting the maturation of the cervix is the presence of the cervical glandular area - hyperechogenic or hypoechogenic layer around the cervical canal. The complete disappearance of this sign is related to the progression of the cervical score and a higher incidence of preterm birth. Grgic and Matijevic described this QGCS (qualitative glandular cervical score) as a good predictor of preterm birth. The studies of Afzali or Asakura also described the absence of this marker as an independent predictor of preterm birth $(1,12-14)$.

Amniotic fluid sludge is a hyperechoic free-floating, matriculate matter in the amniotic fluid close to the internal os of the cervix. Himaya et al. described amniotic fluid sludge as a risk factor of preterm birth and chorioamnionitis (15).

In our studied PPI group, CGA was positive in $21.9 \%$; the same percentage was present for the funneling marker.

Recent scientific publications describe the possibility of predicting preterm delivery using fetal adrenal gland measurements. The fetal adrenal glands are essential in the hormonal materno-fetal signalization of birth. Although the principles of birth initiation were completely described in animals, whereas in humans, the details are still incomplete, especially the exact initiation of birth. The role of 
neuroactive steroids and $\mathrm{CRH}$ (corticoliberin) activation produced in the placenta is typical for primates. CRH stimulates the steroidogenesis in the fetal adrenal glands, and so induces the production of pregnenolone sulfate, DHEAS (dehydroepiandrosterone sulfate) and cortisol $(16,17)$. CRH is the cause of hypertrophy of the central fetal zone of the fetal adrenals. This also emphasizes the fact that the central fetal zone disappears after birth. Finally, steroid production in the fetal zone is one of the signs of true birth initiation (18). It is estimated that during pregnancy, the fetal adrenal glands continually grow, but contrary to other organs, the glands diminish after birth. The weight of the fetal adrenal gland is $3-4 \mathrm{~g}$ at birth, and it is proportionally larger than in adults (19). The growth and size of the fetal adrenals at term is due to the enlargement of the fetal zone. Functionally, the central zone represents zona reticulosa (N.B.: not the medulla), the center of corticosteroid production, and the main source of DHEA for the placenta. Thus, the placental production of estrogens completely depends on the fetal adrenal central zone, and this signalization is one of the principles of the birth initiation $(20,21)$. Van Vuuren created modern volume and reference size charts for fetal kidneys and adrenal glands. In his study, 96 fetuses of physiological pregnancies were recruited and ultrasonographically measured at 4-week intervals. The measured parameters were the length of the kidney and the length of the adrenals (22). In sense of the prediction of preterm birth, the use of these reference tables is questionable because the study, unfortunately, does not describe the central zones and other diameters. In 2012, Ozgüner published a postmortem histopathological morphometric analysis of the adrenal glands and evaluated their weights, volume, width, length, and depth. A significant correlation between the measurements and increasing gestational age and no significant differences between the right and left gland or between the genders were verified (23). As the "in vivo" growth of the adrenals during the pregnancy can occur, this applied only partially.

The fetal zone can be described by transversal ultrasound scan of the fetal abdomen as a hyperechoic structure in the center of another hypoechogenic parenchyma of the gland. Turan describes the adrenals sizes using the 3D ultrasound mode and system of volumetry VOCAL. Finally, he corrected for the values of estimated fetal weight (EFW) as a gestational age-independent parameter. In his study, the growth of the central zone volume over $49.5 \%$ predicted premature birth within 7 days with sensitivity of $100 \%$ and specificity of $89 \%$ as the best predictor. The cAGV (corrected adrenal gland volume) took up the second position with the sensitivity of $81 \%$ and specificity of $87 \%$, while cervicometry with the sensitivity of $56 \%$, and specificity of $60 \%$ took up the third position. An interesting fact is that the growth of the central zone was disproportional, while it was its depth that grew the fastest (3). Another Turan's study compares the 2D ellipsoid measurement of the gland volume with a $3 \mathrm{D}$ calibration of volume. Although the results of these two principles are significant, the conclusion is that the $2 \mathrm{D}$ ellipsoid measurement cannot fully replace the $3 \mathrm{D}$ volume calibration (4). In their 3D study, Ibrahim et al. confirmed Turan's results and also, they described cAGV (corrected adrenal gland volume) and FZE (fetal zone enlargement) as independent predictors of preterm births (24).
Most studies of the fetal adrenal glands versus preterm delivery prediction are prospective and cross-sectional, while describing one group of patients with preterm birth symptoms and signs, and usually not excluding all the factors that could interfere with the adrenal gland growth. Studies usually describe the predictive potential of adrenal gland measurement to predict the preterm birth within 7 days. Lemos et al compare the fetal adrenal gland biometry and cervicometry in preterm delivery in a prospective study of 53 women. The described cut-off value for the depth of the central fetal zone as a predictor for birth within 7 days is $7.2 \mathrm{~mm}$, with the sensitivity of $66.7 \%$ and specificity of $61.8 \%$. These results were postulated as a predictor comparable to cervicometry with a cut-off value of $20 \mathrm{~mm}$ (25). Because the fetal adrenal gland proportions depend on gestational age and individual growth of the fetus, we suppose that it is not possible to define such an absolute cut-off point ( $\mathrm{mm}$ ). Nomograms for absolute measurement of the central zone for each gestational age in physiological pregnancies should be used. However, such reliable nomograms are not available. Nevertheless, the percentage of representation of the fetal zone could be analyzed.

Hoffman's study screened 128 singleton physiological pregnancies with 3D ultrasound volumetry of the whole fetal adrenal gland and gave the following results: there was no significant difference in volumes between the patients who delivered preterm within 7 days, or within more than 14 days. Among the group of women with increased risk of preterm birth, the subgroup of those who delivered prematurely had smaller fetal adrenals as compared to those who did not (26).

In our case-control study of 64 patients, the fetal adrenal gland measurement was provided by meaning the simplest principles, and the following results were obtained. The essential finding was that there was a good possibility for this measurement to be done using standard 2D principles. Out of 66 completely measured patients, only in 2 patients $(3.0 \%)$ it was not possible to verify the fetal adrenal glands (inappropriate acoustic conditions related to high BMI of the patients).

We confirmed a significant correlation $(p=0.02)$ between APAG diameters and gestational age, namely that the latter diameters increased relative to the increase in gestational age. The correlation between circumferences of the whole fetal adrenal gland and the increase in gestational age was more significant than that of the diameter $(\mathrm{p}<0.001)$. This is true evidence of its in vivo continuous growth during the period between the 24th and 37th weeks of gestation. The fact that the adrenal glands are usually of irregular oval shape is the reason why the significance of correlation of circumferences was higher.

The correlation of changes in APFZ and gestational age was not significant $(p=0.06)$, and in this sense, the circumference of the fetal zone did not correlate at all $(\mathrm{p}=0.32)$. The fetal zone increased independently and significantly in other conditions, namely in cases of fetuses delivered preterm.

One of the most important parts of this study was the description of the parameter of "growth" of the fetal zone of the adrenal gland in "AP" as a predictor of preterm birth. The percentage of the central fetal zone revealed significant changes between the patients who delivered prematurely and control groups (PP, PPI-C 
and $\mathrm{C}$ subgroups) $(\mathrm{p}<0.001)$. The differences in changes between patients with imminent preterm birth and physiological control (PPI-C and C control groups) were not significant ( $\mathrm{p}=0.227$ ).

The ideal cut-off value of the proportion of the fetal zone and AP diameter of the whole gland as a marker of preterm delivery up to week 37 was described using the ROC curve.

At the percentage of $45.1 \%$, the sensitivity, specificity, positive predictive value, negative predictive value and likelihood of disease testing negative were $87.5 \%, 85.4 \%, 66.7 \%, 95.3 \%$, and $4.6 \%$, respectively while the confidence intervals of reliability $(95 \%)$ for sensitivity and specificity were $61.6-98.4 \%$ and $72.2-93.9 \%$, respectively.

The proportion of the fetal zone was significantly different between the studied PPI and control groups $(\mathrm{p}<0.001)$, there was also a significant difference between patients with true preterm delivery (PP) and imminent control group (PPI-C) $(\mathrm{p}<0.001)$. On the contrary, the differences between control groups (PPI-C and C) were not significant $(\mathrm{p}=0.227)$.

In conclusion, the aim of our study was to describe the fetal adrenal gland like as a marker that could differentiate the group of the imminent patients who are to deliver prematurely despite standard therapy (tocolytics, magnesium, antibiotics, corticosteroids, etc.). Recently, serious side effects of tocolytics and corticosteroids have been discussed. The use of these additive markers such as fetal adrenal glands can enable a stricter indication of this treatment for a more precisely selected group of truly threatened patients. Fetal adrenal glands measurement could be an additional novel marker usable in distinguishing patients at high risk of preterm delivery. Using simple B-mode ultrasound seems to be reliable enough and makes this method available and technically accessible in routine practice.

\section{References}

1. Yoshimatsu K, Sekiya T, Ishihara K, Fukami T, Otabe T, Araki T. Detection of the cervical gland area in threatened preterm labour using transvaginal sonography in the assessment of the cervical maturation and the outcome of pregnancy. Gynecol Obstetric Invest 2002; 53: 149-156.

2. Romero R, Espinoza J, Kusanovic JP et al. The preterm parturition syndrome. BJOG 2006; 113: 17-42.

3. Turan OM, Turan S, Funai EF et al. Ultrasound measurement of fetal adrenal gland enlargement: an accurate predictor of preterm birth. Am J Obstet Gynecol 2011; 204: 311.e1-311.e10.

4. Turan OM, Turan S, Buhimschi IA et al. Comparative analysis of 2-D versus 3-D ultrasound estimation of the fetal adrenal gland volume and prediction of preterm birth. Am J Perinatol 2012; 29: 673-680.

5. Romero R, Espinoza J, Gonçalves LF, Kusanovic JP, Friel L, Hassan S. The role of inflammation and infection in preterm birth. Semin Reprod Med 2007; 25: 21-25.

6. Hoesli I, Tercanli S, Holzgreve W. Cervical length assessment by ultrasound as a predictor of preterm labour - is there a role for routine screening? Int J Obstet Gynecol 2003; 110: 61-66.

7. Berghella V, Khalifeh A. Ultrasound evaluation of the gravid cervix. In: Norton ME, Scout LM, Feldstein VA, editor. Callen's ultrasonography in obstetrics and gynecology. $6^{\text {th }}$ ed. Elsevier; 2016, p.653-673.
8. Iams JD. Cervical ultrasonography. Ultrasound Obstet Gynecol 1997; 10: 156-160.

9. Leitich, H, Brunbauer M, Kaider A, Egarter CH, Husslein P. Cervical length and dilatation of the cervical os detected by vaginal ultrasonography: A systematic review. Am J Obstet Gynecol 1999; 181: 1465-1472.

10. Mára M, Calda P, Haaková L, Zizka Z, Dohnalová A, Zivný J. Significance of ultrasound vaginal cervicometry in predicting preterm delivery. Med Sci Monit 2002; 8: 72-77.

11. Mella MT, Berhella V. Prediction of preterm birth: cervical sonography. Sem Perinatol 2009; 33: 317-324.

12. Grgic $\mathbf{O}$, Matijevic R, Vasilj $\mathbf{O}$. Qualitative glandular cervical score as a potential new sonomorphological parameter in screening for preterm delivery. Ultrasound Med Biol 2006; 32: 333-338.

13. Afzali N, Mohajeri M, Malek A, Alamatian A. Cervical gland area: a new sonographic marker in predicting preterm delivery. Arch Gynecol Obstet 2012; 285: 255-258.

14. Asakura H, Fukami T, Kurashina R, Tateyama N, Doi D, Takeshita T. Significance of cervical gland area in predicting preterm birth for patients with threatened preterm delivery: comparison with cervical length and fetal fibronectin. Gynecol Obstet Invest 2009; 68: 1-8.

15. Himaya E, Rhalmi N, Girard M et al. Midtrimester intra-amniotic sludge and the risk of spontaneous preterm birth. Am J Perinatol 2011; 28: 815-820.

16. Sirianni R, Mayhew BA, Carr BR, Parker R Jr, Rainey WE. Corticotropin-releasing hormone (CRH) and urocortin act through type one $\mathrm{CRH}$ receptors to stimulate DHEAS production in human fetal adrenal cells. JCEM 2005; 90: 5393-5400.

17. Sirianni R, Rehman KS, Carr BR, Parker R Jr, Rainey WE. Corticotropin-releasing hormone directly stimulates cortisol and the cortisol biosynthetic pathway in human fetal adrenal cells. JCEM 2005; 90: 279-285.

18. McLean M, Smith R. Corticotropin-releasing hormone and human parturition. Reproduction 2001; 121: 493-501.

19. Mesiano S, Jaffe RB. Developmental and functional biology of the primate fetal adrenal cortex. Endocr Rev 1997; 18: 378-403.

20. Rainey WE, Rehman KS, Carr BR. Fetal and maternal adrenals in human pregnancy. Clin Obstet Gynecol 2004; 31: 817-835.

21. Albrecht ED, Aberdeen GW, Pepe GJ. Estrogen elicits cortical zonespecific effects on development of the primate fetal adrenal gland. Endocrinology 2005; 146: 1737-1744.

22. Van Vuuren SH, Damen-Elias HAM, Stigter RH et al. Size and volume charts of fetal kidney, renal pelvis and adrenal gland. Ultrasound Obstet Gynecol 2012; 40: 659-664.

23. Ozgüner G, Sulak O, Koyuncu E. A morphometric study of suprarenal gland development in the fetal period. Surg Radiol Anat 2012; 34: 581-587.

24. Ibrahim MI, Sherif A, El-Kady M et al. Can three-dimensional ultrasound measurement of fetal adrenal gland enlargement predict preterm birth? Arch Gynecol Obstet 2015; 292: 569-578.

25. Lemos AP, Feitosa FEDL, Araujo Júnior EA et al. Delivery prediction in pregnant women with spontaneous preterm birth using fetal adrenal gland biometry. J Matern Fetal Neonatal Med 2016; 29: 3756-3761.

26. Hoffman Sage Y, Lee L, Thomas AM, Benson CB, Shipp TD. Fetal adrenal gland volume and preterm birth: a prospective third-trimester screening evaluation. J Matern fetal Neonatal Med 2016; 29: 1552-1555.

Received March 28, 2021. Accepted April 14, 2021. 